\title{
RESET
}

Recherches en sciences sociales sur Internet

\section{Patrimoine et patrimonialisation numériques}

\section{Francesca Musiani et Valérie Schafer}

\section{(2) OpenEdition}

\section{Journals}

Édition électronique

URL : http://journals.openedition.org/reset/803

DOI : 10.4000/reset.803

ISSN : 2264-6221

\section{Éditeur}

Association Recherches en sciences sociales sur Internet

\section{Référence électronique}

Francesca Musiani et Valérie Schafer, «Patrimoine et patrimonialisation numériques », RESET [En ligne], 6 | 2017, mis en ligne le 18 octobre 2016, consulté le 14 novembre 2019. URL : http:// journals.openedition.org/reset/803; DOI : 10.4000/reset.803

Ce document a été généré automatiquement le 14 novembre 2019.

(c) Association Recherches en sciences sociales sur Internet 


\title{
Patrimoine et patrimonialisation numériques
}

\author{
Francesca Musiani et Valérie Schafer
}

1 La célébration en 2016 des vingt ans de la fondation Internet Archive rappelle à notre attention une initiative précoce de conservation et de mise en patrimoine du numérique : à peine cinq années après les premiers développements du World Wide Web, et alors que celui-ci n'avait pas encore gagné le grand public, le fondateur d'Internet Archive, Brewster Kahle, pensait déjà à son archivage mondial (Mussou, 2012; Masanès, 2006).

2 Cette initiative peut être rattachée au souci des pionniers de l'Internet, universitaires et ingénieurs en premier lieu, de conserver les traces de leurs activités, en particulier techniques. Dès 1969 et les premiers pas du "réseau des réseaux ", les requests for comments (RFCs) - documents de spécification et standardisation technique publiés par l'Internet Engineering Task Force ${ }^{1}$ - constituent le signe de cette mise en mémoire technique. Alexandre Serres souligne d'ailleurs que l'Internet est, à l'origine, largement autoréférentiel et que « le réseau parle du réseau » (Serres, 2000).

3 Mais la démarche de Brewster Kahle est aussi une réaction à ce qui s'est produit pour d'autres médias ; il fait par exemple référence en 1997 à la destruction des films pour la réutilisation de l'argent contenu dans les pellicules (Kahle, 1997). Le sentiment d'une volatilité et d'une fugacité des contenus nativement numériques est ainsi à la racine des premières initiatives (Lyman \& Kahle, 1998).

Des initiatives naissent aussi parmi les chercheurs et universitaires ainsi que les archivistes et bibliothécaires, qui saisissent rapidement que le tournant numérique pose d'importantes questions en termes de conservation. C'est le cas par exemple des projets précoces des pays scandinaves dans la seconde moitié de la décennie 1990 pour étendre le périmètre du dépôt légal, ou encore du projet AOLA lancé au début des années 2000 pour développer un archivage du Web autrichien (AOLA, 2001). Toutes ces démarches font écho aux évolutions que connaît le patrimoine au cours des dernières décennies. Comme le relevait Pierre Nora l'année même de la naissance de la fondation Internet Archive : 
On est passé d'un patrimoine étatique et national à un patrimoine de type social et communautaire où se déchiffre une identité de groupe; et, donc, d'un patrimoine hérité à un patrimoine revendiqué. De matériel et visible, le patrimoine est devenu invisible et symbolique [...] le patrimoine a quitté son âge historique pour entrer dans son âge mémoriel : le nôtre (Nora, 1996).

5 traces de certaines communautés en ligne (Geocities, Mobileme, etc.) par l'Archive Team, l'archivage institutionnel du Web depuis 2006 en France par la Bibliothèque nationale de France (BnF) et l'Institut national de l'audiovisuel (INA), dans le cadre du dépôt légal, ou encore celui de Twitter par la Library of Congress des États-Unis sont autant d'initiatives qui ont contribué à constituer des documents d'emblée numériques en patrimoine. Elles témoignent de la pluralité d'acteurs, aux motivations et enjeux parfois complémentaires, parfois divergents, engagés dans ce processus et surtout d'une inflation documentaire sans précédent. Aux linéaires d'archives des périodes précédentes (Schafer \& Thierry, 2015) répondent les plus de 490 milliards de pages archivées par Internet Archive en vingt années ou encore les 48,5 milliards de versions d'URL conservées par l'Ina en juillet 2016.

S’il est nécessaire de prendre en compte cette abondance sans précédent (Dougherty et al., 2010), la fragmentation, la propagation et la dématérialisation engagent "une rupture épistémologique à la fois pour l'archiviste et le chercheur qui considèrent désormais des 'ressources' ou des 'données' davantage que des documents " (Mussou, 2012 : 264).

La définition du «patrimoine numérique » promue par l'Unesco dans sa Charte sur la conservation du patrimoine numérique (2003), en désignant aussi bien le patrimoine numérisé que des ressources «d'origine numérique » (« qui existent uniquement sous leur forme numérique initiale»), implique de penser le patrimoine numérique tant dans la continuité et la durée que dans la rupture. Elle invite en effet à saisir en quoi le numérique peut faire patrimoine, mais aussi la manière dont le patrimoine est saisi par le numérique, et à prolonger des réflexions en sciences sociales portant sur la «question patrimoniale» (Amougou, 2004) et sur ce que constituent aujourd'hui la notion de patrimoine elle-même et sa « réinvention » (Bourdin, 1984).

Que nous apprennent ces récentes évolutions sur le rapport de nos sociétés à leur patrimoine et au numérique? Que se passe-t-il quand on ne se contente plus de «numériser» la culture mais qu'elle est «d'emblée numérique »? Quelles sont les conséquences sur le patrimoine et sur ses usages - sociaux, culturels, politiques, économiques et scientifiques? Ce numéro présente six articles articulant plusieurs échelles et espaces : mémoire familiale, mémoire collective, micro-histoire, histoire de la Grande guerre, patrimoine scientifique et technique, enjeux mémoriels rattachés à l'histoire de la migration, tensions entre droit à l'oubli et droit à la mémoire, droit d'auteur et enjeux (géo)politiques associés. Au travers de ces contributions, le dossier souhaite éclairer la diversité des politiques de construction, de gestion et de développement du patrimoine numérique, ainsi que quelques acteurs et enjeux des processus de patrimonialisation (Davallon, 2006). 


\section{Patrimoine numérique et héritage social}

9 Les pratiques et processus qui contribuent à la formation et à la reconnaissance du patrimoine numérique engagent des choix, des mises à l'épreuve, un travail social. Ils sont pris en charge par des acteurs variés, et ce à plusieurs échelles. Outre le rôle des professionnels ou celui des passionnés - parfois réunis en associations -, le grand public, les bibliothèques, les musées et des groupes de recherche prennent en charge certains archivages et initient des politiques de préservation. Les entreprises privées, à l'image de Google qui s'est engagé dans la préservation de certains Newsgroups et qui a créé le Google Cultural Institute, participent aussi de ce mouvement, de même que des institutions et forums internationaux, à l'instar de l'Unesco ou de l'International Internet Preservation Consortium (IIPC) ${ }^{2}$.

10 Ces différents acteurs apparaissent au fil de ce numéro parfois de manière isolée, comme le montre un des articles de ce dossier, qui se penche sur une mise en patrimoine d'origine familiale ; cependant, ils apparaissent le plus souvent dans leurs interactions, complémentarités ou encore visions divergentes ou concurrentes de la patrimonialisation numérique. Deux articles du numéro montrent ainsi les enjeux commémoratifs et mémoriels qui s'expriment en ligne, que ce soit autour d'une mémoire davantage " apaisée ", ou d'une mémoire encore vivace, conflictuelle et, dans les deux cas, de mémoires chargées d'enjeux politiques. Aux poids des institutions répondent des initiatives plus locales, individuelles, ou communautaires, et à celles des professionnels de l'histoire, de la documentation, de l'archive ou encore de la communication, celles des amateurs.

11 Choisissant de ne pas opérer de césure étanche entre ces expressions de la mémoire et les tentatives de patrimonialisation ou de réappropriation et diffusion de patrimoines déjà constitués, les auteurs attirent notre attention sur la place des nouveaux acteurs de la «mise en patrimoine», qu'ils soient associatifs, passionnés ou/et militants. Il s'agit là d'une présence perçue avec plus ou moins de bienveillance par les acteurs historiques de la patrimonialisation, qui peuvent en outre se défier des risques de confusion entre le mémorisable et le mémorable (voir Hoog in Coutant \& Stenger, 2010). Ces nouveaux acteurs sont également accusés de promouvoir l'idée illusoire selon laquelle l'appropriation patrimoniale par le public serait rendue possible par la seule mise à disposition. Després-Lonnet le soulignait dès 2009 au sujet de la base Joconde $^{3}$ puis des efforts de mise en ligne du patrimoine culturel européen : «Elle entretient de fait la confusion entre capacité technique de 'rendre accessible' des données, par leur 'mise en ligne' et accès aux savoirs, c'est- à-dire, la possibilité effective donnée à un individu de s'approprier de nouvelles connaissances " (DesprésLonnet, 2009, citant Miège, $1997: 46$ ).

12 Alors que sont posées les questions du rapport à la technique, aux temporalités, à la mémoire individuelle et collective, à l'oubli et au passé, et que sont soulevés les risques de présentisme, ces tendances invitent aussi à reprendre des problématiques déjà parcourues dans le champ des études sur le patrimoine et qui retrouvent de l'actualité : « Devenu un mot-valise, qui produit de l'indifférenciation, le patrimoine est, à bien des égards, dissocié de la relation au passé. En questionnant les modes de représentation du passé dans les processus de patrimonialisation, de conservation, de collection et de médiation, il s'agit tout d'abord d'étudier à nouveaux frais le patrimoine comme mode de relation au passé et au futur» (Colloque Les patrimoines en recherche d'avenir, 
2015). Questionnant ces modes de relation au passé, les travaux dans ce numéro reviennent sur la notion même de patrimoine, dans une approche similaire à celles de Laurajane Smith et Brian Graham. La première a en effet souligné que le patrimoine peut être considéré comme un processus d'engagement davantage qu'une condition (Smith, 2006), tandis que le second le considère tout à la fois comme un vecteur de communication, un moyen de transmettre des idées et valeurs et une connaissance incluant le matériel, l'intangible et le virtuel (Graham, 2002 : 1006).

\section{La fabrique du patrimoine numérique}

L'urgence de la prise en compte de l'émergence et de la «fabrique » d'un patrimoine numérique a été introduite dès 2003 dans la Charte sur la conservation du patrimoine numérique de l'Unesco, soutenue notamment par sa section Communication et information au titre du programme Fostering equitable access to information and knowledge for development et du sous-programme Fostering actions to reduce digital divide and promote social inclusion ${ }^{4}$ (Unesco, 2003; Lusenet, 2007). Elle invite à des recherches portant sur les enjeux et les stratégies d'appropriation et de légitimation du patrimoine numérique, sur ses périmètres de conservation, des études comparatives des politiques et outils de préservation entre différents pays et/ou acteurs (Schafer, Musiani \& Borelli, 2016).

L'aspect peut-être le plus frappant qui émerge à la lecture de ce dossier est l'attention que les auteurs portent à la place des amateurs, des citoyens, des initiatives nées hors des murs des institutions traditionnellement dédiées au patrimoine. Ils éclairent par le terrain et par une observation fine de la fabrique du patrimoine numérique un point encore considéré comme obscur il y a quelques années :

Les projets actuels de patrimonialisation numérique se concentrent surtout sur le

"processus" ou sur le "produit", mais ne considèrent que rarement les

«utilisateurs» [...]. Pour une meilleure interprétation et expérience d'un site

relevant du patrimoine numérique, il nous faut une méthode d'interprétation inclusive, qui devrait tenir compte de la variété de compétences des utilisateurs, dépasser la linéarité de la narration et la subjectivité dans la création des contenus (Rahaman et Tan, 2011, notre traduction).

Si le patrimoine numérique doit être réinscrit dans la longue durée, celle du rapport des sociétés à leur patrimoine, pour en interroger les continuités (Oury, 2012) et les ruptures, la redocumentarisation, la transformation de «traces en communautés de mémoire", aussi bien que les "moyens d'écriture non seulement du soi mais du “nous" » (Le Deuff, 2010) composent toutefois « des archives d'un genre nouveau, à la fois totalement dépendantes des techniques de traçabilité (boutons, imports automatiques, timelines, métadonnées ...), et parfaitement arbitraires, donc non prévisibles » (Merzeau, 2012 : 11).

La valeur patrimoniale que des communautés (Derrot et al., 2012) peuvent attacher, par exemple, à la préservation de groupes de discussions (Usenet, Geocities, etc.), et la volonté de transmission et de patrimonialisation des nouvelles formes de communication et d'expression, méritent ainsi toute notre attention. Au-delà de la question de la valeur patrimoniale des documents et du statut social de l'objet (Davallon, 2006), il convient dès lors de se pencher sur la notion de patrimoine immatériel (Bortolotto, 2011 ; Jadé, 2012), mais aussi celle de bien commun. Mélanie Dulong de Rosnay (2012:143-144) a montré, en s'appuyant sur les travaux d'Elinor Ostrom, en quoi la notion de «bien commun" est au cœur de «créations collectives 
très structurées dont les modalités de gouvernance et d'appropriation appartiennent également aux pairs ».

Comme le notait déjà Maurice Halbwachs (1950), tout groupe organisé crée une mémoire qui lui est propre, et la mémoire individuelle est ancrée dans les processus de remémoration et de localisation propres à des milieux collectifs et communautaires spécifiques. Quand l'Archive Team préserve les pages personnelles de Geocities, c'est un patrimoine " ordinaire », directement dérivé de l'expérience qu'elle prend en charge, à la fois individuel et collectif (Paloque-Berges \& Schafer, 2015 : 258) face à la menace que fait peser sur lui la fermeture du service par Yahoo en 2009.

Dans cet esprit, la conversion des "user generated archives $»^{5}$ en bien commun trouve son achèvement dans l'archivage institutionnel du Web lui-même ${ }^{6}$. (Merzeau, 2012:12).

La mémoire publique «[d]épersonnalisée et dissociée des logiques marchandes qui nous circonscrivent et nous prédisent [...] recrée ici le lien entre individu et communauté politique, dans le temps ouvert d'une réflexivité collective» (Merzeau, 2012) et peut ainsi réconcilier des initiatives communautaires (Derrot et al., 2012) et institutionnelles de nature et périmètres distincts. L'articulation de ces enjeux avec un "marché des données" au sens large, particulièrement foisonnant à l'heure actuelle, reste toutefois nécessaire.

Les initiatives publiques ne font pas oublier que les entreprises du numérique peuvent menacer de disparition des pans entiers de la mémoire numérique collective (à l'instar du cas de Yahoo et Geocities évoqué précédemment). Mais elles affirment aussi leur rôle de premier plan dans la conservation des données et traces numériques (archives de Twitter ; rôle de Google dans le champ du patrimoine numérisé avec Google Books et Google Art Project, ou encore dans la préservation du patrimoine nativement numérique comme l'a montré l'exemple des Newsgroups).

\section{De la navigation à la sédimentation}

[S]i on veut conserver les contenus technologiques, il faut les garder aussi intacts que possibles, en respectant leur intégrité physique, voire en la restaurant, comme on le voit dans les disciplines muséographiques classiques. Mais si on veut conserver des contenus technologiques, il faut les transformer pour permettre leur accessibilité. (Bachimont, $2008: 2$ ).

Le patrimoine né numérique (ou nativement numérique) que nous venons d'évoquer, et que les Anglo-Saxons appellent le Born Digital Heritage ${ }^{7}$, a fait l'objet d'une plus faible attention de la part des chercheurs que le patrimoine converti sous forme numérique (Dufrêne et al., 2013 ; Bachimont, 2014). Cependant, ces différents versants convergent en une patrimonialisation du numérique qui invite le chercheur à considérer non plus seulement la fabrique du patrimoine en tant qu'organisation humaine, mais aussi les « agentivités hybrides » (Abbate, 2012), techniques et humaines, à l'œuvre tout au long de ces processus. Celles-ci mêlent médiations sociales et techniques en un "millefeuille » qui conduit à explorer les boîtes noires, notamment celles de l'archivage du Web, autant que ses modes de gouvernance. Ainsi a-t-on pu montrer la diversité des acteurs engagés dans les processus de patrimonialisation et de leurs motivations, et les dispositifs socio-techniques qu'ils mobilisent pour servir ou illustrer leurs objectifs : le patrimoine numérique devient l'objet-frontière (Star et Griesemer, 1989; Bowker et al., 2016) réclamé par des communautés multiples dont les aspirations et logiques sont tout 
aussi diverses que celles exprimés au sujet du Web "vivant» (Schafer, Musiani \& Borelli, 2016). L'imbrication entre processus techniques et humains qui constituent la fabrique du patrimoine nativement numérique peut être perçue comme un facteur de déstabilisation au sein du monde des archives, des organisations ou encore de la recherche. Cependant, elle invite aussi à se pencher sur les "vracs numériques " (Chabin, 2016) et à retrouver des approches qui peuvent emprunter à des disciplines anciennes, à l'instar de la diplomatique (Chabin, 2011) ou de la philologie (Brügger, 2012).

21 Mais le patrimoine numérisé comme numérique incite aujourd'hui les chercheurs et le monde des archives à se tourner également vers les outils promus par les humanités numériques (Andrews, 2015 ; Le Deuff, 2014 ; Rogers, 2013), que ce soit dans le contexte de la création de corpus, de leur documentation ou encore de leur exploitation ${ }^{8}$. Plusieurs articles de ce dossier abordent la question des métadonnées et celle de l'indexabilité au cœur des pratiques de numérisation du patrimoine comme du patrimoine nativement numérique. Louise Merzeau relevait déjà l'importance de cette question: les métadonnées associées aux contenus patrimoniaux, loin de seulement décrire des contenus, peuvent permettre leur segmentation et recomposition. Ainsi " [p]ar sa tendance à s'autoréférencer dès son émission, l'information numérique emporte avec elle son archivage et son accessibilité » (Merzeau, 2012: 4). Accessibilité et interopérabilité sont des problématiques qui aujourd'hui impliquent une diversité d'acteurs, chercheurs et ingénieurs de recherche, professionnels de la conservation, entreprises.

Enfin, une réflexion sur un autre patrimoine, que l'on pourrait qualifier de "patrimoine $\mathrm{du}$ numérique» (matériels, périphériques, documents d'accompagnement, etc.) s'impose également pour retrouver des environnements numériques complexes dont les contenus ne constituent que la face archivée de l'iceberg. Cette réflexion semble tout aussi nécessaire que celle menée sur les contenus, d'autant qu'elle est rarement portée par les mêmes services ou acteurs, à quelques exceptions près comme la conservation du jeu vidéo et celle de ses supports matériels et guides.

\section{Présentation du numéro}

Les six articles et l'introduction qui composent ce dossier n'échappent pas à l'interdisciplinarité des études qui portent sur le patrimoine numérique, car ils mettent ici en dialogue des analyses relevant du champ historique, des sciences de l'information et de la communication, de la sociologie de l'innovation, des humanités numériques ou encore des sciences juridiques. Les auteurs nous font pénétrer des univers différents : on passe ainsi de la mise en ligne et en patrimoine des archives familiales du joueur de hockey professionnel canadien Léo Gravelle, explorée avec une minutie anthropologique par Fannie Valois-Nadeau, à la dimension européenne des commémorations de la Première Guerre mondiale et du corpus associé à leur analyse qu'aborde Enrico Natale; nous découvrons des sites Web dédiés à la mémoire de l'immigration maghrébine qu'étudie Sophie Gebeil, mais aussi les enjeux transnationaux que posent les débats autour du droit à l'oubli ou du droit d'auteur, auxquels nous introduisent respectivement Mélanie Dulong de Rosnay \& Andrés Guadamuz et Rolf Weber \& Lennart Chrobak. Au cours de cette déambulation, on croise 
aussi des communautés, à l'instar des pionniers des réseaux qui laissent un riche patrimoine scientifique et technique, via leurs communications en ligne, sur lesquelles s'est penchée Camille Paloque-Berges. Le patrimoine numérisé fait davantage l'objet des études de Fannie Valois-Nadeau et Rolf Weber et Lennart Chrobak, alors que le patrimoine nativement numérique est au cœur des corpus de Sophie Gebeil, Enrico Natale et Camille Paloque-Berges.

Articulant réflexions sur les données, les traces, les archives, le patrimoine, la mémoire et l'histoire, mais aussi les dimensions internationales et plus locales, plusieurs articles entrelacent également les réflexions scientifiques et celles d'ordre épistémologique et méthodologique (notamment dans les articles de Camille Paloque-Berges et Enrico Natale) et nous éclairent autant sur les évolutions du patrimoine que celles du numérique.

Si les publics, les usages ou encore la dimension économique sont moins présents dans les analyses, ils apparaissent toutefois en filigrane à l'heure d'une culture " amateur " qui donne lieu à des modalités de "réception créative ", appuyée sur l'acquisition de compétences et sur des formes d'engagements et de mobilisations inédits (Flichy, 2010). Car il sera souvent question, au fil de ces pages, d'initiatives d'amateurs et d'hybridation entre pratiques professionnelles et en amateur par acquisition, imitation, motivation. Les professionnels de l'archivage ou des musées, ainsi que les chercheurs, ne seront toutefois pas absents de ces univers s'imbriquant en un monde commun, bien qu'hétérogène. Dominique Boullier préconisait à cet égard qu'une politique du patrimoine et de la mémoire puisse s'appuyer à la fois sur la capacité des communautés à produire leur mémoire, comme le fait la tradition, la capacité à réviser ces mémoires et les capitaliser, comme le fait l'activité scientifique, et enfin la capacité, propre aux médias, à faire émerger de nouveaux centres d'intérêt et références (Boullier, 2008).

De la fabrique d'un patrimoine familial à celle renouvelée de la mémoire de l'immigration maghrébine ou de la Grande Guerre en passant par le patrimoine scientifique et technique des utilisateurs pionniers d'Internet, l'étude des formes, productions, pratiques, discours et idéologies qui accompagnent ces initiatives permet de mieux cerner les contours d'un patrimoine numérique et d'un patrimoine du numérique en cours de formation, de même que leurs dimensions sociales, politiques ou encore culturelles.

\section{BIBLIOGRAPHIE}

ABBATE Janet (2012). «L'histoire de l'Internet au prisme des STS », Le temps des médias, 18, pp. 170-180.

Amougou Emmanuel (2004). « Les sciences sociales et la question patrimoniale », in Amougou Emmanuel, Chadoin Olivier, Fayolle Lussac Bruno, Girard Paulette, Godier Patrice, Gurov Radian \& Kocher André, La Question patrimoniale : De la " patrimonialisation » à l'examen des situations concrètes. Paris, L'Harmattan. 
ANDREWS Tara (2015). Digital Humanities, Living Book [en ligne], URL : http:// www.livingbooksabouthistory.ch/fr/book/digital-humanities

Aola (2001) Austrian On-Line Archive, page d'accueil [en ligne], URL : http:// www.ifs.tuwien.ac.at/ aola/

BACHIMONT Bruno (2014). Patrimoine et numérique : technique et politique de la mémoire, Paris, INA.

BACHIMONT Bruno (2008). « La conservation du patrimoine numérique : enjeux et tendances », edossiers de l'audiovisuel « Patrimoine numérique : mémoire virtuelle, mémoire commune? ", Ina expert [en ligne], URL : http://www.ina-expert.com/e-dossier-de-l-audiovisuel-patrimoinenumerique-memoire-virtuelle-memoire-commune/la-conservation-du-patrimoine-numeriqueenjeux-et-tendance.html

BOULLIER Dominique (2008). « Politiques de la mémoire en temps d'incertitude », e-dossiers de l'audiovisuel « Patrimoine numérique : mémoire virtuelle, mémoire commune ? », Ina expert [en ligne], URL : http://www.ina-expert.com/e-dossier-de-l-audiovisuel-patrimoine-numeriquememoire-virtuelle-memoire-commune/politiques-de-la-memoire-en-temps-d-incertitude.html BOURDIN Alain (1984). Le patrimoine réinventé, Paris, PUF.

Bowker Geoffrey C., Timmermans Stefan, CLARKe Adele E. \& BALKa Ellen (eds., 2016). Boundary Objects and Beyond. Working with Leigh Star, Cambridge, MA : The MIT Press.

BORTOLOTTO Chiara (2011). Le patrimoine culturel immatériel : Enjeux d'une nouvelle catégorie. Paris, Editions de la Maison des Sciences de l'Homme.

BRÜGGER Niels (2012). « L'historiographie des sites Web : quelques enjeux fondamentaux », Le Temps des médias, 18, pp. 159-169.

CAROU Alain (2003). «Quel avenir pour un patrimoine numérique?», 1895. Mille huit cent quatrevingt-quinze, 41, pp. 209-215. [Republié en ligne], URL : http://1895.revues.org/813

CHABIN Marie-Anne (2016). « Réflexion sur le vrac numérique », Impressions, expressions. Le blog de Marie-Anne Chabin. [En ligne], URL : http://www.marieannechabin.fr/2016/05/reflexion-surle-vrac-numerique/

CHABIN Marie-Anne (2011). « Peut-on parler de diplomatique numérique », Impressions, expressions. Le blog de Marie-Anne Chabin. [En ligne], URL : http://www.marieannechabin.fr/ diplomatique-numerique/

COLLOQUE « LES PATRIMOINES EN RECHERCHE D’AVENIR », [En ligne], URL : http:// colloquepatrimoines.passes-present.eu/

COUTANT Alexandre \& STENGER Thomas (2010). « Pratiques et temporalités des réseaux socionumériques : logique de flux et logique d'archive », MEI - Médiation et information, 32, pp. 125-136.

DAVALLON Jean (2006). Le don du patrimoine : Une approche communicationnelle de la patrimonialisation. Paris, Hermès sciences publications.

Derrot Sophie, Fauduet Louise, Oury Clément \& Peyrard Sébastien (2012). “Preservation Is Knowledge : A community-driven preservation approach", 9th International Conference on Preservation of Digital Objects (iPRES), Canada.

DESPRÉS-LONNET Marie (2009). « L'écriture numérique du patrimoine, de l'inventaire à l'exposition : Les parcours de la base Joconde », Culture \& Musées, 14, pp. 19-38. 
DufRene Bernadette, IHADJADENE Madjid \& BRUCKMANN Denis (dir.) (2013). Numérisation du patrimoine: Quelles médiations ? Quels accès? Quelles cultures ?, Paris, Hermann.

Dougherty Meghan, Meyer Eric, MadSEn Christine, VAn den heuvel Charles, Thomas Arthur \& WYATT Sally (2010). Researcher Engagement with Web Archives : State of the Art, London, JISC.

Dulong DE RoSNAY Mélanie \& MUSIANI Francesca (2012). « The Preservation of Digital Heritage : Epistemological and Legal Reflections », ESSACHESS - Journal for Communication Studies, 5 (2), pp. 81-94.

DULONG DE ROSNAY Mélanie (2011). « Réappropriation des données et droit à la rediffusion », Hermès, 59, pp. 65-66.

DULONG DE ROSNAY Mélanie (2012). "Réseaux de production collaborative de connaissances », in Letonturier Eric (ed.), Les Réseaux, Paris, CNRS éditions, coll. « Les essentiels d'Hermès », pp. 141-146.

Fuichy Patrice (2010). Le sacre de l'amateur. Sociologie des passions ordinaires à l'ère numérique, Paris, Le Seuil.

GRAHAM Brian (2002). “Heritage as Knowledge : Capital or Culture ?”, Urban Studies, 33, pp. 1003-1017.

HALBWACHS Maurice (1950). La mémoire collective, Paris : Albin Michel.

JADÉ Mariannick (2012). Le patrimoine immatériel : Perspectives d'interprétation du concept de patrimoine, Paris, L'Harmattan.

KAHLE Brewster (1997). « Preserving the Internet », Scientific American, [En ligne], URL : http:// www.scientificamerican.com/article/preserving-the-internet/

LE DEUFF Olivier (2010). « Quelles mnémotechniques pour l'Internet?», in Pignier Nicole \& Lavigne Michel (eds.), revue MEI, n³2, « Mémoires \& Internet », Paris, L'Harmattan, 41-51. LE DEUFF Olivier (dir.) (2014). Le temps des humanités digitales. La mutation des sciences humaines et sociales, Limoges, FYP éditions.

LUSENET Yola (2007). "Tending the Garden or Harvesting the Fields : Digital Preservation and the UNESCO Charter on the Preservation of the Digital Heritage”, Library Trends, 56 (1), pp. 164-182.

LYMAN Peter \& KAHLE Brewster (1998). “Archiving Digital Cultural Artifacts. Organizing an Agenda for Action”, D-Lib Magazine, [En ligne], URL : http://mirror.dlib.org/dlib/july98/07lyman.html

MASANES Julien (2006). Web Archiving, Berlin Heidelberg, Springer.

MERZEAU Louise (2012). « Faire mémoire des traces numériques », e-dossiers de l'audiovisuel «Sciences humaines et sociales et patrimoine numérique », Ina expert, [En ligne], URL : http:// www.ina-expert.com/e-dossier-de-l-audiovisuel-sciences-humaines-et-sociales-et-patrimoinenumerique/faire-memoire-des-traces-numeriques.html

MIÈGE Bernard (1997). La Société conquise par la communication : 2. La communication entre industrie et espace public, Grenoble, Presses universitaires de Grenoble.

Mussou Claude (2012). «Et le Web devint archive : enjeux et défis », Le Temps des Médias, 19, pp. 259-266.

NoRA Pierre (1996). « Préface », in Sire Marie-Anne, La France du patrimoine, Les choix de la mémoire, Paris, Gallimard/MONUM. 
OURY Clément (2012). « Une simple adaptation ? L’héritage du dépôt légal face à la mutation numérique », Implications philosophiques, [En ligne], URL : http://www.implications-

philosophiques.org/actualite/une/une-simple-adaptation-lheritage-du-depot-legal-face-a-lamutation-numerique/

PALOQUe-BERges Camille \& SCHAFER Valérie (2014). « Les archives des réseaux numériques :

périmètres, enjeux, défis ", Culture et Recherche, 129, pp. 68-69.

PALOQUe-BERges Camille \& SCHAFER Valérie (2015). « Quand la communication devient patrimoine », Hermès, 71, pp. 255-262.

RAHAMAN Hafizur \& TAN Beng-Kiang (2011). “Interpreting Digital Heritage: A Conceptual Model With End-Users' Perspective”, International Journal of Architectural Computing, 9 (1), pp. 99-113.

RoGERs Richard (2013). Digital Methods, Cambridge, MA, The MIT Press.

SCHAFER Valérie, MUSIANI Francesca \& BORELli Marguerite (2016). "Negotiating the Web of the Past", French Journal for Media Research, 6, [En ligne], URL : http://frenchjournalformediaresearch.com/ lodel/index.php?id=952

SCHAFER Valérie \& THIERRY Benjamin (2015). «L'ogre et la Toile. Le rendez-vous de l'histoire et des archives du Web », Socio, 4, pp. 75-96.

SERRES Alexandre (2000). Aux sources d'Internet : l'émergence d'ARPANET. Thèse de doctorat, Université Rennes 2, [En ligne], URL : https://halshs.archives-ouvertes.fr/tel-00312005/ document

SMITH Laurajane (2006). Uses of Heritage, London \& New York, Routledge.

STAR Susan Leigh \& GRIESEMER James (1989). “Institutional Ecology, 'Translations’ and Boundary Objects: Amateurs and Professionals in Berkeley's Museum of Vertebrate Zoology, 1907-39”, Social Studies of Science, 19 (3), pp. 387-420.

UNESCO (2003). Charte sur la conservation du patrimoine numérique, [En ligne], URL : http:// portal.unesco.org/fr/ev.php-URL_ID=17721\&URL_DO=DO_TOPIC\&URL_SECTION=201.html

\section{NOTES}

1. Groupe international, informel et en principe ouvert à tout individu, communiquant en ligne, qui produit encore aujourd'hui la plupart des standards de l'Internet.

2. Espace d'échange dédié à l'archivage du Web né en 2003. http://www.netpreserve.org

3. Base de données répertoriant les collections des musées français, créée en 1975 et consultable sur Minitel avant de passer sur le Web. Elle est gérée par le ministère de la Culture

4. UNESCO (2003). Records of the General Conference, 32nd session, Paris, 2003. http:// unesdoc.unesco.org/images/0013/001331/133171e.pdf\#page $=80$

5. Expression modelée sur celle des User-generated content (contenus générés par les utilisateurs), fréquemment utilisée à propos du Web 2.0.

6. En France, celui-ci a été programmé en 2006 par la loi DADvSI, qui a chargé la BnF et l'Ina d'assurer le dépôt légal des publications en ligne relevant du périmètre national.

7. Le projet AOLA note dès 2001 l'existence de cette expression : http:// www.ifs.tuwien.ac.at/ aola/publications/trans10

8. Nous pensons par exemple au développement d'outils, fonctionnalités et d'interfaces spécifiques de fouille de données et métadonnées par la BnF et l'Ina pour les fonds constitués au 
moment des attentats parisiens de janvier et novembre 2015 que nous avons suivis au sein du projet ASAP (Archives Sauvegarde Attentats Paris), mené en partenariat avec ces deux institutions et financé par le CNRS (https://asap.hypotheses.org, site visité le 14 septembre 2016), ou encore à l'implémentation de la recherche plein texte dans les archives du Web des années 1990 par la BnF, exploitée dans le cadre de notre projet Web90 (projet ANR-14-CE29-0012).

\section{AUTEURS}

\section{FRANCESCA MUSIANI}

CNRS/Paris-Sorbonne/UPMC, Institut des sciences de la communication

\section{VALÉRIE SCHAFER}

CNRS/Paris-Sorbonne/UPMC, Institute des sciences de la communication 